FIT 62 years, $\mathrm{p}=0.10)$ and gender $(\mathrm{p}=0.41)$. Although the differences did not reach significance, advanced pathology in the FIT group was much higher, both in polyps $\geq 10 \mathrm{~mm}$ diameter (FIT $86 \%$ v no FIT $14 \%, \mathrm{p}=0.30$ ) and CRC incidence (FIT 4.5\% v no FIT 1.0\%, p = 0.19).

Conclusions Despite the challenges of managing clinical services during a pandemic, our data demonstrates an increased use of FIT in the colorectal pathway in line with updated guidance. This is particularly encouraging as there was insufficient time for an adequate communication strategy. However, a substantial proportion of patients were referred based on FIT results with neither NG12 nor DG30 compliant symptoms; perhaps showing that FIT can potentially increase referrals if used incorrectly. Some patients were referred with a negative FIT and a preponderance of NG12 symptoms, possibly indicating that clinical concern over traditional cancer symptoms may prompt referral despite an objectively very low cancer risk.

\section{PTH-98 FAECAL IMMUNOCHEMICAL TEST, FAST SCORE OR NG12 CRITERIA FOR DETECTION OF CANCER}

${ }^{1}$ Rigers Cama*, ${ }^{1}$ Neel Kapoor, 'Lefkothea Zacharopoulou, 'Leila Mebarek, 'Haroon Bhatti, ${ }^{2}$ Philip Sawyer, ${ }^{3}$ Bharat Patel, ${ }^{1} J o n a t h a n ~ L a n d y . ~{ }^{1}$ Gastroenterology Dept, West Hertfordshire Hospitals NHS Trust, Watford, WD18 OHB; ${ }^{2}$ Parkbury House Surgery, Herts Valleys CCG, St Albans, AL1 3HD; ${ }^{3}$ Chemical Pathology Dept, West Hertfordshire Hospitals NHS Trust, Watford, WD18 OHB

\subsection{6/gutjnl-2021-BSG.301}

Introduction Studies have evaluated FIT in patients meeting NG12 criteria suggesting greater accuracy for colorectal cancer (CRC) detection. The FAST score (faecal-Hb, age and sex test score) was proposed to improve the utility of $\mathrm{fHb}$ in the diagnosis of CRC. In 2019, Herts Valleys CCG instituted the use of FIT for patients in primary care presenting with symptoms meeting DG30 and some lower risk NG12 criteria (PPV $<3 \%$ ), excluding those with higher risk symptoms of iron deficiency anaemia (IDA), mass or rectal bleeding. We aimed to evaluate the utility of FIT with NG12 referral criteria and FAST score for the detection of CRC in our population.

Methods The medical records of all patients undertaking a FIT sample with a minimum of 6 months follow up between June 2019 and July 2020 were reviewed and cross referenced with the trust cancer database. Other outcomes recorded included inflammatory bowel disease and high-risk adenomas (defined as polyps of $\geq 1 \mathrm{~cm}, \geq 5$ polyps or high-grade dysplasia). FIT analysis was performed using a single OC-Sensor analyser (Eiken Chemical Co., Tokyo, Japan). FAST scores (> 2.12 versus $<2.12$ ) were calculated as previously described by Digby et al. (2019). Sensitivity, specificity, predictive values and numbers needed to investigate, were calculated using MedCalc ${ }^{\circledR}$ statistical software.

Results 3460 patients returned a FIT sample. The median age of population was 66 (IQR 56-76), with 57\% being female. 1046 patients underwent any investigation with 701 patients having full colonic evaluation. 22\% had FIT $>10 \mu \mathrm{g} / \mathrm{g}, 75 \%$ had FAST score $>2.12$ and 59\% met NG12 criteria. Sensitivity for CRC, in FIT, FAST group (> 2.12) and NG12 groups was 94\% (95\% CI 84-99\%), 100\% (95\% CI 93-100\%) and $82 \%$ (95\% CI 67-91\%) respectively. Specificity for CRC was $83 \%$ (95\% CI 82-84\%), 25\% (95\% CI 24-27\%) and $42 \%$ (95\% CI 40-43\%) respectively. The number needed to investigate to detect a patient with cancer was 16,52 and 50 for
FIT $>10 \mu \mathrm{g} / \mathrm{g}, \quad$ FAST score $>2.12$ and NG12 criteria respectively.

Conclusions FAST score $>2.12$ has the best sensitivity for detection of cancer. However, the specificity is low and significantly more patients would require investigation. FIT $>10$ $\mu \mathrm{g} / \mathrm{g}$ performed better than NG12 criteria.

\section{PTH-99 FAECAL IMMUNOCHEMICAL TESTSFOR YOUNGER PATIENTS PRESENTING WITH BOWEL SYMPTOMS}

${ }^{1}$ Lefkothea Zacharopoulou*, ${ }^{1}$ Rigers Cama, ${ }^{1}$ Neel Kapoor, 'Leila Mebarek, ${ }^{1}$ Haroon Bhatti, ${ }^{2}$ Philip Sawyer, ${ }^{3}$ Bharat Patel, 'Jonathan Landy. ${ }^{1}$ Gastroenterology Dept, West Hertfordshire Hospitals NHS Trust, Watford, WD18 OHB; ${ }^{2}$ Parkbury House Surgery, Herts Valleys CCG, St Albans, AL1 3HD; ${ }^{3}$ Chemical Pathology Dept, West Hertfordshire Hospitals NHS Trust, Watford, WD18 OHB

\subsection{6/gutjnl-2021-BSG.302}

Introduction Quantitative faecal immunochemical tests (FIT) are recommended by NICE (DG30) guidelines for use in patients with suspected colorectal cancer in primary care. However, the utility of FIT in patients under the age of 50 versus the use of faecal calprotectin is unclear. In 2019, Herts Valleys CCG instituted the use of FIT for patients over the age of 40 years, presenting with symptoms meeting DG30 and some lower risk NG12 criteria, excluding those with higher risk symptoms of iron deficiency anaemia (IDA), mass or rectal bleeding. We aim to evaluate the accuracy of FIT for significant bowel disease (SBD) in patients under 50 years with those 50-59 and over 60 years in our population.

Methods The medical records of all patients undertaking a FIT sample with a minimum of 6 months follow up between June 2019 and July 2020 were reviewed. The outcome of SBD (a composite of either colorectal cancer, inflammatory bowel disease or high-risk adenomas (defined as polyps of $\geq 1 \mathrm{~cm}, \geq 5$ polyps or high-grade dysplasia) was recorded. FIT analysis was performed using a single OC-Sensor io analyser (Eiken Chemical Co., Tokyo, Japan). The sensitivity, specificity, predictive values and accuracy of FIT for SBD were assessed for each age group. Fisher's exact test was used to assess the differences in sensitivity and specificity of FIT between patients $<50$ and older age groups. MedCalc ${ }^{\circledR}$ statistical software was used for all calculations.

Results 3460 patients with bowel symptoms undertook a FIT sample. 132 patients had SBD. 13\% of patients were $<50$ with a FIT result $\geq 10 \mu \mathrm{g} / \mathrm{g}$ in $12 \%$. $22 \%$ were $50-59$ with a FIT result $\geq 10 \mu \mathrm{g} / \mathrm{g}$ in $16 \%$. $65 \%$ were $\geq 60$ with a FIT result $\geq 10 \mu \mathrm{g} / \mathrm{g}$ in $26 \%$. The sensitivity, specificity, PPV, NPV and accuracy of a FIT result $\geq 10 \mu \mathrm{g} / \mathrm{g}$ for patients 50-59 were $85 \%$ (CI 66 to 95.8\%), 87\% (CI 84 to 89\%), 19\% (CI $15.8 \%$ to $23 \%$ ), $99.4 \%$ (CI $98.5 \%$ to $99.8 \%$ ) and $87 \%$ (CI $84 \%$ to $89 \%$ ) and for patients $\geq 60$ years were $86 \%$ (CI 77 to $92 \%$ ), $77 \%$ (CI 75 to $78.5 \%$ ), $14 \%$ (CI 13 to $16 \%$ ), $99.2 \%$ (CI $98.7 \%$ to $99.5 \%$ ) and $77 \%$ (CI $75 \%$ to $79 \%$ ). The sensitivity, specificity, PPV, NPV and accuracy of a FIT result $\geq 10 \mu \mathrm{g} / \mathrm{g}$ for patients $<50$ years were $87.5 \%$ (CI 47 to 99.7\%) $\mathrm{p}=1.0,89 \%$ (CI 85.7 to $91.9 \%$ ) $\mathrm{p}=0.35$ and $<0.01$, $13 \%$ (CI 9 to $17.8 \%$ ), $99.7 \%$ (CI 98.4 to $99.96 \%$ ) and $89 \%$ (CI 86 to $92 \%$ ) respectively.

Conclusion FIT performed well for the detection of SBD in all age groups with equivalent sensitivity between age groups and improved specificity in younger age groups compared with patients $\geq 60$ years. 